\title{
On filling minimality of simple Finsler manifolds
}

\author{
Henrik Koehler
}

\begin{abstract}
This paper states a formula for the difference of the HolmesThompson volumes of two simple Finsler manifolds of arbitrary dimension, in terms of the difference of the boundary distances and their derivatives. An application is a conditioned result on filling minimality.
\end{abstract}

\section{Introduction}

Let $M$ be a smooth compact manifold with boundary $\partial M$ and a reversible Finsler metric $F$. Then $(M, F)$ is called simple, if it is convex, without conjugate points, and any two points $x, y \in M$ are connected by a unique geodesic segment. Simple manifolds are known to be contractible, and whether a manifold is simple can be determined from the data of boundary distances (see [3]; the change from Riemannian to Finsler metrics has no consquence).

In this article, $(M, F)$ shall be called minimal filling (for Finsler volume) if $\operatorname{vol}_{\tilde{F}}(\tilde{M}) \geq \operatorname{vol}_{F}(M)$ holds for all oriented Finsler manifolds $(\tilde{M}, \tilde{F})$ with $\partial \tilde{M}=$ $\partial M$ and $\operatorname{dist}_{\tilde{F}}(y, z) \geq \operatorname{dist}_{F}(y, z)$ for all $y, z \in \partial M$, where vol denotes the HolmesThompson (symplectic) volume. The notion of filling volume was originally introduced in [6] in the context of systolic and isoperimetric inequalities. It should be mentioned that the Holmes-Thompson volume coincides with the standard volume in the Riemannian case; hence the above notion comprises filling minimality for Riemannian manifolds.

An open question is whether simple manifolds are minimal fillings. In contrast, a manifold that contains regions which are not (or are too sparsely) intersected by minimal geodesics between boundary points clearly cannot be a minimal filling. Therefore, some restriction has to be imposed on $(M, F)$ to guarantee that the data of boundary distances give sufficient information about the interior of $M$; here simplicity seems a adequate requirement.

In the Riemannian case, the question of filling minimality is often considered together with the boundary rigidity question, which asks whether a Riemannian

Mathematics Subject Classification (2010): 53C23, 53C60, 53C70.

Keywords: Minimal fillings, geometric inverse problems, boundary distances. 
metric is determined (up to isometries) from its boundary distances. Filling minimality was proved for conformal metrics and for two-dimensional Riemannian SGM-manifolds (see [4]), and for metrics close to one another in a $C^{3, \alpha}$-sense (see [5]). In two recent articles ([1] and [2]), the problems of boundary rigidity and filling minimality were solved for simple Riemannian metrics close to the flat (resp., hyperbolic) metric in a $C^{2}$-sense (resp., $C^{3}$-sense). Also, filling minimality was recently shown for two dimensional Finsler metrics with minimal geodesics (see [7]). Further, a local result was obtained in [8], stating volume monotonicity with respect to boundary distance increasing changes of the Finsler metric in a $C^{\infty}$-neighbourhood for simple Finsler manifolds of any dimension.

Corollary 3.2 states that an inequality for the boundary distances of two simple Finsler manifolds implies the same inequality between the symplectic volumes,

if the dimension is $n=2$ (as already known from [7]);

or $n=3$ or $n=4$ and the sum of the boundary distances is again

a boundary distance function of some simple Finsler manifold;

or the boundary distance functions are $C^{2}$-close to each other.

It should be noticed that the third condition differs from results like Proposition 1.2 in [5] or Theorem 2 in [8] on volume monotonicity, since it pertains to $C^{2}$-small changes for the boundary distance function itself, rather than perturbations of the underlying Riemann or Finsler metric. To clarify what " $C^{2}$-close" means for boundary distances, their behaviour near the diagonal is examined in Section 4. One might ask, whether the second condition is necessary; however, Proposition 5.1 shows, that for $n=3$, the sum of boundary distance functions need not come from a simple Finsler manifold.

The essential tool is a relationship between the canonical symplectic two-form on the cotangent bundle and boundary distances (cf. [9]). This allows the boundary integral in Santaló's formula to be expressed in terms of the mixed second derivative of the boundary distance function (see Proposition 2.2). Using this identity, Proposition 3.1 expresses the difference of Finsler volumes as an integral of the difference of boundary distances; thereby it generalizes what was known for two-dimensional Riemannian manifolds (Theorem 1.4 of [4]).

Acknowledgements. The author is grateful to Sergei Ivanov for his comments on a prior preprint version. He also kindly provided a proof of the $C^{1,1}$-regularity of the exponential map along the zero section in the Finsler case (Proposition 4.1). Further, the author would like to thank Christopher Croke, Gerhard Knieper and the referee for their helpful remarks.

\section{Santaló-type integral formulas}

In all that follows, only simple Finsler manifolds are considered. Since these are always contractible, one may restrict to the model case of an $n$-disk.

Henceforth, let $B=\left\{x \in \mathbb{R}^{n}:\|x\|<1\right\}$ denote the unit ball, let $S^{n-1}$ be its boundary and let $\bar{B}=B \cup S^{n-1}$ be its closure. Suppose $\bar{B}$ is equipped with 
a reversible Finsler metric $F: T \bar{B} \rightarrow[0, \infty)$, i.e., $F$ is a norm on every $T_{x} \bar{B}$, depending smoothly on $x \in \bar{B}, F(-v)=F(v)$ for all $v \in T \bar{B}$, and the bilinear form associated to $F$ at $w \in T_{x} \bar{B} \backslash\{0\}$ via

$$
g_{w}(u, v):=\left.\frac{d^{2}}{2 d s d t}\right|_{s=t=0} F^{2}(w+s u+t v) \quad\left(u, v \in T_{x} \bar{B}\right),
$$

is positive definite on $T_{x} \bar{B}$. For later use, notice also that $g_{w}(w, w)=F^{2}(w)$ and $g_{r w}=g_{w}$ for all $r \neq 0$. Further, let $\ell: \bar{B} \times \bar{B} \rightarrow[0, \infty)$ denote the length metric induced by $F$; that is, $\ell(x, y)=\inf _{c} \int F(\dot{c})$, where $c$ ranges over all smooth curves connecting $x$ with $y$. Throughout, $(\bar{B}, F)$ is required to be a simple Finsler manifold.

For $v \in T \bar{B}$, let $\gamma_{v}:\left[t_{-}(v), t_{+}(v)\right] \rightarrow \bar{B}$ be the maximal geodesic with $\dot{\gamma}_{v}(0)=v$, so $\gamma_{v}\left(t_{ \pm}(v)\right) \in S^{n-1}$. Thus, the geodesic flow on the unit tangent bundle $S \bar{B}:=$ $\{v \in T \bar{B}: F(v)=1\}$ is given by

$$
\Phi:\left\{(v, t) \in S \bar{B} \times \mathbb{R}: t_{-}(v) \leq t \leq t_{+}(v)\right\} \rightarrow S \bar{B}, \quad(v, t) \mapsto \phi^{t}(v)=\dot{\gamma}_{v}(t) .
$$

Moreover, let $\Gamma:=\left\{v \in S \bar{B}: \pi(v) \in S^{n-1}, t_{+}(v)>0\right\}$ be the set of inward pointing unit vectors over the boundary, where $\pi: T \bar{B} \rightarrow \bar{B}$ denotes the footpoint projection. Since $(\bar{B}, F)$ is simple, $t_{+}: \Gamma \rightarrow(0, \infty)$ is smooth, and

$$
\Phi:\left\{(v, t): v \in \Gamma, t \in\left(0, t_{+}(v)\right)\right\} \rightarrow S B
$$

is an orientation preserving diffeomorphism.

On $T \bar{B} \backslash\{0\}$, there is a natural one-form $\theta$, called the Hilbert form:

$$
T_{w} T \bar{B} \ni \xi \mapsto \theta_{w}(\xi)=g_{w}(w, D \pi(w) \xi)
$$

It comes from the canonical one-form on $T^{*} \bar{B}$ via the Legendre transform; consequently, $d \theta$ is a symplectic two-form (cf. [10], page 26), and $\theta \wedge(d \theta)^{n-1}$ defines a volume form on $S \bar{B}$. In fact, it is related to the Liouville form $\lambda$ via

$$
\lambda=c_{n} \theta \wedge(d \theta)^{n-1}, \quad \text { where } \quad c_{n}:=\frac{(-1)^{n(n+1) / 2+1}}{(n-1) !},
$$

Hence, integration with respect to the Holmes-Thompson volume yields

$$
\int_{\bar{B}} f d \operatorname{vol}=\frac{c_{n}}{\operatorname{vol}\left(S^{n-1}\right)} \int_{S \bar{B}} f \circ \pi \theta \wedge(d \theta)^{n-1} \quad \forall f \in C(\bar{B}) .
$$

Both $d \theta$ and $\lambda$ are invariant with respect to the geodesic flow (see [10], Section 5.4).

Now, a Finsler version of Santaló's formula reads:

Lemma 2.1. For every function $f \in L^{1}(S \bar{B}, \lambda)$, there holds

$$
\int_{S \bar{B}} f \lambda=c_{n} \int_{\Gamma} \int_{0}^{t_{+}} f \circ \phi^{t} d t(d \theta)^{n-1} .
$$


Proof. For $v \in S \bar{B}$ and $t \in\left(t_{-}(v), t_{+}(v)\right)$, fix some $(\xi, \tau) \in T_{(v, t)}(S \bar{B} \times \mathbb{R})$. Then there holds $\Phi^{*} \lambda_{(v, t)}=c_{n} \cdot\left(\left(\phi^{t}\right)^{*} \theta+d t\right) \wedge(d \theta)^{n-1}$, because $\left(\phi^{t}\right)^{*} d \theta=d \theta$ and

$$
\begin{aligned}
\Phi^{*} \theta(\xi, \tau) & =\theta_{\phi^{t}(v)}(D \Phi(v, t)(\xi, \tau))=\theta_{\phi^{t}(v)}\left(D \phi^{t}(v) \xi+\tau \frac{d}{d t} \phi^{t}(v)\right) \\
& =\left(\phi^{t}\right)^{*} \theta(\xi)+\tau g_{\phi^{t}(v)}\left(\phi^{t}(v), \frac{d}{d t} \pi \circ \phi^{t}(v)\right)=\left(\left(\phi^{t}\right)^{*} \theta+d t\right)(\xi, \tau) .
\end{aligned}
$$

Hence, the claimed identity is obtained from the transformation formula.

Further, set $S^{n-1} \times S^{n-1} \backslash$ diagonal =: $\Pi$ for brevity. Then the map

$$
\psi: \Gamma \rightarrow \Pi, \quad \psi(u)=\left(\pi(u), \exp \left(t_{+}(u) u\right)\right)
$$

is a diffeomorphism with respect to the orientation induced by $\psi$. This allows the integral in Lemma 2.1 to be expressed in the following form:

Proposition 2.2. The integral of any $f \in C(S \bar{B})$ can be computed via

$$
\int_{S \bar{B}} f d \lambda=c_{n} \int_{\Pi} \int_{0}^{\ell} f \circ \phi^{t} \circ \psi^{-1} d t\left(d_{1} d_{2} \ell\right)^{n-1} .
$$

Proof. Set $V=\exp ^{-1}(\bar{B}) \subset T \bar{B}$ and consider the map

$$
\Psi: V \rightarrow \bar{B} \times \bar{B}, \quad \Psi(w)=(\pi(w), \exp (w)),
$$

which is related to $\psi$ via $\psi(u)=\Psi\left(t_{+}(u) u\right)$, for $u \in \Gamma$. Since all geodesics minimize distance, the first variation formula yields that

$$
d_{1} \ell(x, \exp (w)) v=\frac{-g_{w}(w, v)}{F(w)} \quad \forall x \in \bar{B}, v, w \in T_{x} \bar{B}, w \in V \backslash\{0\}
$$

Therefore,

$$
\Psi^{*}\left(d_{1} \ell\right)_{w}=\pi^{*} d_{1} \ell(\pi(w), \exp (w))=\frac{-\theta_{w}}{F(w)}
$$

Now, given $u \in \Gamma, \xi \in T_{u} \Gamma$, consider a smooth curve $u:(-\varepsilon, \varepsilon) \rightarrow \Gamma$ with $u(0)=u$, $\dot{u}(0)=\xi$ and set $w(s)=t_{+}(u(s)) u(s)$, so that $\psi \circ u=\Psi \circ w$.

From equation $(2.1)$ and $g_{u}=g_{w}$ one infers

$$
\begin{aligned}
-\psi^{*}\left(d_{1} \ell\right)(\xi) & =-\Psi^{*}\left(d_{1} \ell\right)_{w}(\dot{w}(0))=\frac{1}{F(w)} \theta_{w}(\dot{w}(0)) \\
& =\frac{1}{t_{+}(u)} g_{w}\left(t_{+}(u) u,\left.\frac{d}{d s}\right|_{0} \pi \circ w(s)\right)=g_{u}(u, D \pi(u) \dot{u}(0))=\theta(\xi) .
\end{aligned}
$$

Using " $d=d_{1}+d_{2}$ ", one concludes that $d \theta=-d \psi^{*} d_{1} \ell=\psi^{*} d_{1} d_{2} \ell$. Finally, Proposition 2.2 follows from Lemma 2.1 and the transformation formula. 
To illustrate the geometric nature of $D_{1,2}^{2} \ell$, consider $x, y \in \bar{B}, y \neq x$, and let $u=\exp _{x}^{-1}(y) / \ell(x, y)$ be the (Finsler) unit vector at $x$ pointing towards $y$ and

$$
P_{u}: T_{x} \bar{B} \rightarrow T_{x} \bar{B}, \quad P_{u} v=v-g_{u}(u, v) u
$$

the projection onto the $g_{u}$-orthogonal complement of $u$. Then there holds:

Proposition 2.3. The mixed second derivative of $\ell(x, y)$ satisfies

$$
D_{1,2}^{2} \ell(x, y)(v, w)=\frac{-g_{u}\left(P_{u} v, D \exp _{x}^{-1}(y) w\right)}{\ell(x, y)} \quad \forall v \in T_{x} \bar{B}, w \in T_{y} \bar{B} .
$$

Proof. Let $c:(-\varepsilon, \varepsilon) \rightarrow \bar{B} \backslash\{x\}$ a smooth curve with $c(0)=y$ and $\dot{c}(0)=w$ and set $r(t)=\ell(x, c(t))$; hence one can write $c(t)=\exp _{x}(r(t) u(t))$ with $F(u(t))=1$ for all $t$. Again $d_{1} \ell(x, c(t)) v=-g_{u(t)}(u(t), v)$, from the first variation formula, so

$$
-D_{1,2}^{2}(x, y)(v, w)=\left.\frac{d}{d t}\right|_{0} g_{u(t)}(u(t), v)=\left.\frac{d^{2}}{2 d s d t}\right|_{0} F(u(t)+s v)^{2}=g_{u}(v, \dot{u}(0)) .
$$

On the other hand, $P_{u} D \exp _{x}^{-1}(y) w=P_{u}(\dot{r}(0) u(0)+r(0) \dot{u}(0))=\ell(x, y) \dot{u}(0)$, because $g_{u}(u, \dot{u}(0))=\left.\frac{d}{2 d t}\right|_{0} F(u(t))^{2}=0$.

Remarks. For $x, y \in S^{n-1}$ and $w \in T_{y} S^{n-1}$, one has $u(t)=\psi^{-1}(x, c(t))$, so $\dot{u}(0)=D_{2} \psi^{-1}(x, y) w$ and thus $-D_{1,2}^{2} \ell(x, y)(v, w)=g_{\psi^{-1}(x, y)}\left(v, D_{2} \psi^{-1}(x, y) w\right)$. Further, if $\left(\xi_{1}, \ldots, \xi_{n-1}, v_{1}, \ldots, v_{n-1}\right)$ are local coordinates on $\Pi$, the coordinate expression of $\left(d_{1} d_{2} \ell\right)^{n-1}$ reads

$$
\left(d_{1} d_{2} \ell\right)^{n-1}=\left(\sum_{i, j=1}^{n} \frac{\partial^{2} \ell}{\partial \xi_{i} \partial v_{j}} \cdot d \xi_{i} \wedge d v_{j}\right)^{n-1}=\operatorname{det}\left(\frac{\partial^{2} \ell}{\partial \xi_{i} \partial v_{j}}\right) \cdot(d \xi \wedge d v)^{n-1}
$$

where $d \xi \wedge d v:=d \xi_{1} \wedge d v_{1}+\cdots+d \xi_{n-1} \wedge d v_{n-1}$. In particular, nondegeneracy of $d \theta$ implies that the determinant does not vanish.

\section{An application to filling minimality}

The Santaló-type integral formula from Proposition 2.2 can be used to obtain an equality between volume differences and a certain integral of differences of boundary distances. Again, set $\Pi=S^{n-1} \times S^{n-1} \backslash$ diagonal.

Proposition 3.1. Suppose $(\bar{B}, F)$ and $(\bar{B}, \tilde{F})$ are simple Finsler manifolds with induced distances $\ell$ and $\tilde{\ell}$, respectively. Then for the related Holmes-Thompson volumes, there holds

$$
\operatorname{vol}_{\tilde{F}}(\bar{B})-\operatorname{vol}_{F}(\bar{B})=\frac{c_{n}}{\operatorname{vol}\left(S^{n-1}\right)} \int_{\Pi}(\tilde{\ell}-\ell) \sum_{k=0}^{n-1}\left(d d_{2} \tilde{\ell}\right)^{k} \wedge\left(d d_{2} \ell\right)^{n-1-k} .
$$


Proof. Taking $f \equiv 1$ in Proposition 2.2, one obtains

$$
\operatorname{vol}_{F}(\bar{B})=\frac{\operatorname{vol}_{F}(S \bar{B})}{\operatorname{vol}\left(S^{n-1}\right)}=\frac{c_{n}}{\operatorname{vol}\left(S^{n-1}\right)} \int_{\Pi} \ell\left(d_{1} d_{2} \ell\right)^{n-1} .
$$

Subtracting this from the corresponding expression for $\tilde{F}$ gives

$$
\operatorname{vol}_{\tilde{F}}(\bar{B})-\operatorname{vol}_{F}(\bar{B})=\frac{c_{n}}{\operatorname{vol}\left(S^{n-1}\right)} \int_{\Pi} \tilde{\ell}\left(d d_{2} \tilde{\ell}\right)^{n-1}-\ell\left(d d_{2} \ell\right)^{n-1} .
$$

The integrand can be decomposed as

$$
\begin{gathered}
\tilde{\ell}\left(d d_{2} \tilde{\ell}\right)^{n-1}-\ell\left(d d_{2} \ell\right)^{n-1}=(\tilde{\ell}-\ell)\left(d d_{2} \tilde{\ell}\right)^{n-1}+\ell\left(\left(d d_{2} \tilde{\ell}\right)^{n-1}-\left(d d_{2} \ell\right)^{n-1}\right) \\
=(\tilde{\ell}-\ell)\left(d d_{2} \tilde{\ell}\right)^{n-1}+\ell d d_{2}(\tilde{\ell}-\ell) \wedge\left(\sum_{k=0}^{n-2}\left(d d_{2} \tilde{\ell}\right)^{k} \wedge\left(d d_{2} \ell\right)^{n-2-k}\right) .
\end{gathered}
$$

Writing $\eta=\sum_{k=0}^{n-2}\left(d d_{2} \tilde{\ell}\right)^{k} \wedge\left(d d_{2} \ell\right)^{n-2-k}$ for simplicity, $\eta=1$ for $n=2$, while for $n>2, \eta$ is an exact $2(n-2)$-form of degree $n-2$ in each factor of $S^{n-1} \times S^{n-1}$. Also, using " $d=d_{1}+d_{2}$ " and " $d_{i}^{2}=0$ ", one obtains

$$
\ell d d_{2}(\tilde{\ell}-\ell)-(\tilde{\ell}-\ell) d d_{2} \ell=\ell d d_{2} \tilde{\ell}-\tilde{\ell} d d_{2} \ell=d\left(\ell d_{2} \tilde{\ell}+\tilde{\ell} d_{1} \ell\right)-d_{2} \ell \wedge d_{2} \tilde{\ell}+d_{1} \ell \wedge d_{1} \tilde{\ell}
$$

Because $d_{1} \ell \wedge d_{1} \tilde{\ell} \wedge \eta$ and $d_{2} \ell \wedge d_{2} \tilde{\ell} \wedge \eta$ have degree $n$ in the first and second variable, respectively, they cancel. For simplicity, set

$$
\left(d d_{2} \tilde{\ell}\right)^{n-1}+d d_{2} \ell \wedge \eta=\sum_{k=0}^{n-1}\left(d d_{2} \tilde{\ell}\right)^{k} \wedge\left(d d_{2} \ell\right)^{n-1-k}=: \hat{\eta} .
$$

One infers from the above decomposition that

$$
\operatorname{vol}_{\tilde{F}}(\bar{B})-\operatorname{vol}_{F}(\bar{B})=\frac{c_{n}}{\operatorname{vol}\left(S^{n-1}\right)} \int_{\Pi}(\tilde{\ell}-\ell) \hat{\eta}+d\left(\ell d_{2} \tilde{\ell} \wedge \eta\right)+d\left(\tilde{\ell} d_{1} \ell \wedge \eta\right) .
$$

Here, $\hat{\eta}$ is integrable, because $\hat{\eta}=n \cdot \int_{0}^{1}\left(d d_{2}((1-a) \ell+a \tilde{\ell})\right)^{n-1} d a$ holds pointwise on $\Pi$, and the integrability of $\left(d d_{2}((1-a) \ell+a \tilde{\ell})\right)^{n-1}$ will be satisfied $^{1}$ in Corollary 4.5.

Now, let $U(\varepsilon):=\{(x, y) \in \Pi: \ell(x, y)<\varepsilon\}$ denote a tubular $\varepsilon$-neighbourhood of $\partial \Pi=\operatorname{diag}\left(S^{n-1} \times S^{n-1}\right)$. Then Stokes' theorem implies

$$
\int_{\Pi \backslash U(\varepsilon)} d\left(\ell d_{2} \tilde{\ell} \wedge \eta\right)=\int_{\partial U(\varepsilon)} \ell d_{2} \tilde{\ell} \wedge \eta=\varepsilon \int_{\partial U(\varepsilon)} d_{2} \tilde{\ell} \wedge \eta=\varepsilon \int_{\Pi \backslash U(\varepsilon)} d d_{2} \tilde{\ell} \wedge \eta .
$$

\footnotetext{
${ }^{1}$ In fact, since two-forms can be muted without invoking sign changes, $\hat{\eta}$ can be considered a homogenous polynomial in $d d_{2} \ell$ and $d d_{2} \tilde{\ell}$ with all coefficients equal to 1 . The claimed integral representation thus follows from the binomial expansion and the fact that

$$
n \cdot\left(\begin{array}{c}
n-1 \\
k
\end{array}\right) \int_{0}^{1} a^{k}(1-a)^{n-1-k} d a=1 \quad \forall k \in\{0, \ldots, n-1\} .
$$
}


However, $d d_{2} \tilde{\ell} \wedge \eta=\hat{\eta}-\left(d d_{2} \ell\right)^{n-1}$, so

$$
\lim _{\varepsilon \rightarrow 0} \int_{\Pi \backslash U(\varepsilon)} d d_{2} \tilde{\ell} \wedge \eta=\int_{\Pi} \hat{\eta}-\int_{\Pi}\left(d d_{2} \ell\right)^{n-1} \quad \text { and } \quad \lim _{\varepsilon \rightarrow 0} \varepsilon \int_{\Pi \backslash U(\varepsilon)} d d_{2} \tilde{\ell} \wedge \eta=0 .
$$

Likewise with $\tilde{U}(\varepsilon):=\{(x, y) \in \Pi: \tilde{\ell}(x, y)<\varepsilon\}$

$$
\int_{\Pi \backslash \tilde{U}(\varepsilon)} d\left(\tilde{\ell} d_{1} \ell \wedge \eta\right)=\varepsilon \int_{\Pi \backslash \tilde{U}(\varepsilon)} d d_{1} \ell \wedge \eta=-\varepsilon \int_{\Pi \backslash \tilde{U}(\varepsilon)} d d_{2} \ell \wedge \eta \stackrel{\varepsilon \rightarrow 0}{\longrightarrow} 0 .
$$

Thus, the integrals of the exact forms cancel, and one obtains the claimed equality.

Corollary 3.2. Let $(B, F)$ and $(B, \tilde{F})$ be simple and such that $\ell(y, z) \leq \tilde{\ell}(y, z)$ holds for all $y, z \in S^{n-1}$. Then $\operatorname{vol}_{F}(\bar{B}) \leq \operatorname{vol}_{\tilde{F}}(\bar{B})$ with equality implying $\ell(y, z)=$ $\tilde{\ell}(y, z)$ for all $y, z \in S^{n-1}$, provided one of the following criteria is satisfied:

1. The dimension is $n=2$; or $n \leq 4$ and there is a simple Finsler metric $\bar{F}$ having boundary distances $\bar{\ell}=\tilde{\ell}+\ell$.

2. $\tilde{\ell}$ lies in an appropriate $C^{2}$-neighbourhood of $\ell$.

Proof. Conditions 1 and 2 guarantee that $\hat{\eta}$ is a volume form, so Proposition 3.1 implies the assertions:

For $n=2$, Proposition 2.2 states that $\hat{\eta}=d d_{2} \ell+d d_{2} \tilde{\ell}$ corresponds to the sum of volume forms on the unit inward tangent bundle over $\partial B$, hence is again a volume form. Then $\hat{\eta}$ can be decomposed as

$$
\begin{array}{ll}
\hat{\eta}=\frac{1}{2}\left(d d_{2} \ell\right)^{2}+\frac{1}{2}\left(d d_{2}(\ell+\tilde{\ell})\right)^{2}+\frac{1}{2}\left(d d_{2} \tilde{\ell}\right)^{2} & \text { for } n=3 \text { and } \\
\hat{\eta}=\frac{2}{3}\left(d d_{2} \ell\right)^{3}+\frac{1}{3}\left(d d_{2}(\ell+\tilde{\ell})\right)^{3}+\frac{2}{3}\left(d d_{2} \tilde{\ell}\right)^{3} & \text { for } n=4 .
\end{array}
$$

Here, the mixed term is a volume form, if $\ell+\tilde{\ell}$ is simple.

For the second condition: for every $\varepsilon>0$, there is a constant $\delta>0$ such that

$$
\left|\left(d d_{2} \ell\right)^{n-1}(x, y)\right| \geq \delta\left|(d x \wedge d y)^{n-1}\right| \quad \text { and } \quad \sup \left\|D_{1,2}^{2} \ell(x, y)\right\| \leq \frac{1}{\delta}
$$

for all $(x, y) \in \Pi$ with $\|x-y\| \geq \varepsilon$. Accordingly, the expansion

$$
\begin{aligned}
\hat{\eta} & =\sum_{k=0}^{n-1}\left(d d_{2} \ell\right)^{n-1-k} \wedge\left(d d_{2} \ell+d d_{2}(\tilde{\ell}-\ell)\right)^{k} \\
& =\sum_{i=0}^{n-1} \sum_{k=i}^{n-1}\left(\begin{array}{l}
k \\
i
\end{array}\right)\left(d d_{2} \ell\right)^{n-1-i} \wedge\left(d d_{2}(\tilde{\ell}-\ell)\right)^{i}
\end{aligned}
$$

shows that $\hat{\eta}$ is dominated by the term $n\left(d d_{2} \ell\right)^{n-1}$ as long as $\left\|D_{1,2}^{2}(\tilde{\ell}-\ell)\right\|$ is smaller than some constant depending on $\delta$ and $n$. Since $\varepsilon$ was arbitrary, this yields a $C^{2}$-neighbourhood for $\ell$; see, however, the following Remark 2. 
Remarks. 1) The first condition could be generalized for $n>4$. Namely, one can choose $a_{i} \in[0,1]$ and $c_{i}>0$, such that $\hat{\eta}=\sum_{i} c_{i}\left(d d_{2}\left(a_{i} \ell+\left(1-a_{i}\right) \tilde{\ell}\right)\right)^{n-1}$. Then $\hat{\eta}$ is a volume form, if $\left(1-a_{i}\right) \ell+a_{i} \tilde{\ell}$ are boundary distances of simple Finsler metrics $F_{i}$.

2) Boundedness of $\left\|D_{1,2}^{2}(\tilde{\ell}-\ell)\right\|$ would require that $D_{1,2}^{2} \tilde{\ell}(x, y)$ and $D_{1,2}^{2} \ell(x, y)$ have the same asymptotic behaviour as $y \rightarrow x$; so $\tilde{F}$ and $F$ a priori would have to coincide on $S^{n-1}$, as was pointed out by S. Ivanov. Namely, due to Proposition 2.3, $D_{1,2}^{2} \ell$ becomes singular along the diagonal; indeed the scaling depends on the direction. To avoid this deficiency, one can consider another criterion for positivity of $\hat{\eta}$ on $\{(x, y) \in \Pi:\|x-y\|<\varepsilon\}$, for $\varepsilon$ small. Actually, in local coordinates $(\xi, v)$,

$\hat{\eta}=n \int_{0}^{1}\left(d d_{2}((1-a) \ell+a \tilde{\ell})\right)^{n-1} d a=n \int_{0}^{1} \operatorname{det}\left(\frac{\partial^{2}((1-a) \ell+a \tilde{\ell})}{\partial \xi_{i} \partial v_{j}}\right) d a \cdot(d \xi \wedge d v)^{n-1}$.

Thus, it is sufficient to ensure that $\operatorname{det}\left((1-a) \frac{\partial^{2} \ell}{\partial \xi_{i} \partial v_{j}}+a \frac{\partial^{2} \tilde{\ell}}{\partial \xi_{i} \partial v_{j}}\right)$ does not vanish for $a \in(0,1)$. In view of the remark after Proposition 2.3, this is satisfied, provided $\tilde{\psi}$ lies in a suitable $C^{1}$-neighbourhood of $\psi$ and $\tilde{g}_{\tilde{\psi}^{-1}(x, y)}$ is sufficiently close to $g_{\psi^{-1}(x, y)}$, for $(x, y) \in \Pi$. In the remark after Proposition 4.4, such a condition is stated in terms of $\ell$ and $\tilde{\ell}$.

\section{Analysis of $D_{1,2}^{2} \ell$ near the diagonal}

Starting from Proposition 2.3, the objective of this section is to find two-sided estimates for $D_{1,2}^{2} \ell(x, y)$ as $x$ tends to $y$, in order to control the singularity of $\left(d_{1} d_{2} \ell\right)^{n-1}$ on the diagonal.

First, since $F$ is a Finsler metric, there is a constant $C_{1}>1$, such that

$$
\frac{1}{C_{1}^{2}}\|v\|^{2} \leq g_{u}(v, v) \leq C_{1}^{2}\|v\|^{2} \quad \forall u, v \in T_{x} \bar{B}, u \neq 0
$$

where $\|v\|$ denotes the standard Euclidean norm on $\mathbb{R}^{n}$. Furthermore, $C_{1}$ can be chosen to be independent of $x$, for compactness of $\bar{B}$. As a consequence, one infers for the related distances

$$
\frac{1}{C_{1}}\|x-y\| \leq \ell(x, y) \leq C_{1}\|x-y\| \quad \forall x, y \in \bar{B} .
$$

The term $D \exp _{x}^{-1}(y)$ requires some scrutiny: On a Finsler manifold, the exponential map at any point is known to be a local $C^{1}$-diffeomorphism on a neighbourhood of the origin, but of class $C^{\infty}$ only away from zero (see [10], Theorem 11.1.1). $\mathrm{S}$. Ivanov mentioned that the regularity is in fact $C^{1,1}$ :

Proposition 4.1. Let $(N, F)$ a smooth Finsler manifold without boundary. Then for every point $p \in N$, the differential $D \exp _{p}$ of the exponential map is Lipschitzcontinuous at $0 \in T_{p} N$. 
Proof (by S. Ivanov). In local coordinates on a neighbourhood of $p$, the Finsler metric $F$ can be considered a function $F_{1}(x, v)$ of points $x \in \mathbb{R}^{n}$ and vectors $v \in \mathbb{R}^{n}$. For simplicity, one can assume that $x(p)=0$ and extend $F_{1}$ arbitrarily to a smooth Finsler metric on the entire $\mathbb{R}^{n}$. Define a family $F_{t}, t \in \mathbb{R}$ of "blow-ups" of the metric $F_{1}$ by $F_{t}(x, v)=F_{1}(t x, v)$. This is a smooth family of metrics, so it defines a smooth family of exponential maps $E_{t}: \mathbb{R}^{n} \rightarrow \mathbb{R}^{n}$ (here $E_{t}$ is the exponential map at 0 of the metric $F_{t}$ ). More precisely, this family is smooth on any compact set separated away from the origin. We consider it in a neighborhood of the unit sphere. As $\left(\mathbb{R}^{n}, F_{0}\right)$ is a Minkowski space, $E_{0}$ is the identity, so its second derivative is zero. Since $D^{2} E_{t}$ depends smoothly on $t$, there exists a constant $C>0$ such that, for $|t|$ small enough, $\left\|D^{2} E_{t}(v)\right\| \leq C|t|$ at any point $v$ of the unit sphere (here $\|\cdot\|$ is a norm on bilinear forms). Because $F_{1}(t x, t v)=|t| \cdot F_{t}(x, v)$, the map $x \mapsto t x$ is a constant stretch and thus transfers geodesics in $\left(\mathbb{R}^{n}, F_{t}\right)$ to geodesics in $\left(\mathbb{R}^{n}, F_{1}\right)$. Consequently, $E_{1}(v)=t E_{t}(v / t)$, so $D E_{1}(v)=D E_{t}(v / t)$ and $D^{2} E_{1}(v)=\frac{1}{t} D^{2} E_{t}(v / t)$ for all $t \neq 0$. Rescaling back to the original metric, we get $\left\|D^{2} E_{1}(v)\right\| \leq C$ for all $v$ on the sphere of radius $t>0$. So $D^{2} E_{1}$ is bounded near the origin, hence $E_{1}=\exp _{0}$ is of class $C^{1,1}$.

Notice that, because of the smooth dependence of the generating vector field on the geodesic flow with respect to changes in the Finsler metric, the corresponding maps $D^{2} E_{t, p}: S^{n-1} \rightarrow\left(\mathbb{R}^{n} \otimes \mathbb{R}^{n}\right)^{*}$ vary smoothly with $t$ and $p \in N$. Therefore, the Lipschitz constant $C$ can be chosen in a way that depends continuously on $p$. This allows for a uniform estimate in the next lemma:

Lemma 4.2. There exists a constant $C_{2}>1$, such that for all $x \neq y \in \bar{B}$ with $\|x-y\|<1 / C_{2}$ and all $v \in T_{x} \bar{B}, w \in T_{y} \bar{B}$, there holds

$$
\left|\ell(x, y) \cdot D_{1,2}^{2} \ell(x, y)(v, w)+g\left(P_{u} v, w\right)\right| \leq C_{2}\|x-y\| \cdot\|w\| \sqrt{g_{u}\left(P_{u} v, v\right)} .
$$

Proof. When extending $F$ to a neighbourhood of $\bar{B}$, Proposition 4.1 guarantees the existence of some $C_{3}>0$, such that $\left\|D \exp _{x}(\tilde{v})-\mathbf{1}\right\| \leq C_{3}\|\tilde{v}\|$ for all $\tilde{v} \in T_{x} \bar{B}$ with $\|\tilde{v}\|<1 / C_{3}$; and again $C_{3}$ can be selected independently of $x$, since $\bar{B}$ is compact. If $\|\tilde{v}\|<1 /\left(2 C_{3}\right)$, then the inverse of $D \exp _{x}(\tilde{v})$ satisfies

$$
\left\|D \exp _{x}(\tilde{v})^{-1}-\mathbf{1}\right\| \leq \frac{\left\|D \exp _{x}(\tilde{v})-\mathbf{1}\right\|}{1-\left\|D \exp _{x}(\tilde{v})-\mathbf{1}\right\|} \leq 2 C_{3}\|\tilde{v}\|,
$$

where the first inequality follows from

$$
\left\|\left(A^{-1}-\mathbf{1}\right) w\right\| \leq\|A-\mathbf{1}\| \cdot\left\|A^{-1} w\right\| \leq\|A-\mathbf{1}\| \cdot\left(\|w\|+\left\|\left(A^{-1}-\mathbf{1}\right) w\right\|\right) .
$$

Taking $\tilde{v}=\exp _{x}^{-1}(y)$ in the above estimate, one obtains that

$$
\left\|D \exp _{x}^{-1}(y)-\mathbf{1}\right\| \leq 2 C_{1}^{2} C_{3} \cdot\|x-y\|
$$

as long as $\|x-y\|<1 /\left(2 C_{1}^{2} C_{3}\right)$, because, due to (4.1) and (4.2),

$$
\left\|\exp _{x}^{-1}(y)\right\| \leq C_{1} \cdot F\left(\exp _{x}^{-1}(y)\right)=C_{1} \cdot \ell(x, y) \leq C_{1}^{2}\|x-y\| .
$$


Now, applying the Cauchy inequality to the formula from Proposition 2.3 yields

$$
\begin{array}{r}
\left|\ell(x, y) \cdot D_{1,2}^{2} \ell(x, y)(v, w)+g\left(P_{u} v, w\right)\right|=\left|g_{u}\left(P_{u} v,\left(D \exp _{x}^{-1}(y)-\mathbf{1}\right) w\right)\right| \\
\leq \sqrt{g_{u}\left(\left(D \exp _{x}^{-1}(y)-\mathbf{1}\right) w,\left(D \exp _{x}^{-1}(y)-\mathbf{1}\right) w\right)} \cdot \sqrt{g_{u}\left(P_{u} v, v\right)}
\end{array}
$$

for all $v \in T_{x} \bar{B}, w \in T_{y} \bar{B}$. According to (4.3), the first factor satisfies

$$
\begin{aligned}
\sqrt{g_{u}\left(\left(D \exp _{x}^{-1}(y)-\mathbf{1}\right) w,\left(D \exp _{x}^{-1}(y)-\mathbf{1}\right) w\right)} & \leq C_{1}\left\|\left(D \exp _{x}^{-1}(y)-\mathbf{1}\right) w\right\| \\
& \leq 2 C_{1}^{3} C_{3}\|x-y\| \cdot\|w\|
\end{aligned}
$$

provided that $\|x-y\| \leq 1 /\left(2 C_{1}^{2} C_{3}\right)$, which proves the assertion.

Restricting to the case of $x, y \in S^{n-1}$, let $e_{x y} \in T_{x} S^{n-1}$ denote the Euclidean unit vector tangent to the shortest arc on $S^{n-1}$ that connects $x$ with $y$. Then $T_{x} S^{n-1}$ allows a decomposition into $\mathbb{R} \cdot e_{x y}$ and $T_{x y}:=T_{x} S^{n-1} \cap T_{y} S^{n-1}$, its orthogonal complement with respect to the Euclidean scalar product $\langle\cdot, \cdot\rangle$. The following estimates for $g_{u}\left(P_{u} v, w\right)$ will be needed in the sequel.

Lemma 4.3. There exists a constant $C_{4}>C_{2}$ such that there hold

$$
\begin{aligned}
g_{u}\left(P_{u} e_{x y}, e_{x y}\right) & \leq C_{4}\|x-y\|^{2} \geq g_{u}\left(P_{u} e_{y x}, e_{y x}\right), \\
g_{u}\left(P_{u} v, v\right) & \geq \frac{1}{C_{4}}\|v\|^{2} \quad \forall v \in T_{x y}
\end{aligned}
$$

whenever $x, y \in S^{n-1}$ satisfy $0 \neq\|x-y\|<1 / C_{4}$.

Proof. First, when integrating (4.3) from the proof of Lemma 4.2, one obtains

$$
\begin{aligned}
\left\|y-x-\exp _{x}^{-1}(y)\right\| & =\left\|\int_{0}^{1}\left(1-D \exp _{x}^{-1}(t y+(1-t) x)\right)(y-x) d t\right\| \\
& \leq \int_{0}^{1} 2 C_{1}^{2} C_{3}\|t y-t x\| \cdot\|x-y\| d t=C_{1}^{2} C_{3}\|x-y\|^{2}
\end{aligned}
$$

if $\|x-y\| \leq 1 /\left(2 C_{1}^{2} C_{2}\right)$. On the other hand, one infers from plane geometry that

$$
\left\|\frac{x-y}{\|x-y\|}-e_{y x}\right\|=\left\|\frac{y-x}{\|x-y\|}-e_{x y}\right\|=2 \sin (s / 4) \leq\|x-y\|,
$$

where $s=2 \arcsin (\|x-y\| / 2)$ is the Euclidean length of the shortest arc between $x$ and $y$ on $S^{n-1}$. One concludes from the triangle inequality that

$$
\left\|\frac{\exp _{x}^{-1}(y)}{\|x-y\|}-e_{x y}\right\| \leq\left(1+C_{1}^{2} C_{3}\right)\|x-y\| .
$$

Since $P_{u}\left(\exp _{x}^{-1}(y) /\|x-y\|\right)=0$, one can apply (4.1) and (4.4) to get

$$
\begin{aligned}
g_{u}\left(P_{u} e_{x y}, e_{x y}\right) & =g_{u}\left(P_{u}\left(e_{x y}-\frac{\exp _{x}^{-1}(y)}{\|x-y\|}\right), P_{u}\left(e_{x y}-\frac{\exp _{x}^{-1}(y)}{\|x-y\|}\right)\right) \\
& \leq C_{1}^{2}\left\|\frac{\exp _{x}^{-1}(y)}{\|x-y\|}-e_{x y}\right\|^{2} \leq C_{1}^{6} C_{3}^{2}\|x-y\|^{2} .
\end{aligned}
$$


Similar reasoning shows the same estimate for $e_{y x}$, thereby verifying the first two claimed inequalities.

Next, let $z \in \mathbb{R}^{n}$ be the unique vector such that $g_{u}(v, z)=\langle v, y-x\rangle$ for all $v \in \mathbb{R}^{n}$. Since $\langle v, y-x\rangle=0$ for all $v \in T_{x y}$, a Bessel inequality yields

$$
1=g_{u}(u, u) \geq \frac{g_{u}(u, v)^{2}}{g_{u}(v, v)}+\frac{g_{u}(u, z)^{2}}{g_{u}(z, z)} \Rightarrow g_{u}\left(P_{u} v, v\right) \geq g_{u}(v, v) \frac{g_{u}(u, z)^{2}}{g_{u}(z, z)} .
$$

For the numerator, the Cauchy inequality and (4.4) and (4.2) show

$$
\begin{aligned}
g_{u}(u, z) & =\left\langle\frac{\exp _{x}^{-1}(y)}{\ell(x, y)}, y-x\right\rangle=\frac{\|y-x\|^{2}-\left\langle y-x-\exp _{x}^{-1}(y), y-x\right\rangle}{\ell(x, y)} \\
& \geq \frac{\|x-y\|^{2}-\|x-y\| \cdot\left\|y-x-\exp _{x}^{-1}(y)\right\|}{\ell(x, y)} \\
& \geq \frac{\|x-y\|^{2}\left(1-C_{1}^{2} C_{2}\|x-y\|\right)}{\ell(x, y)} \geq \frac{\|x-y\|}{2 C_{1}} .
\end{aligned}
$$

Further, (4.1) implies a similar inequality for the dual metric $g_{u}^{*}$, so $g_{u}(z, z)=$ $g_{u}^{*}\left((y-x)^{T},(y-x)^{T}\right) \leq C_{1}^{2}\|x-y\|^{2}$ in the denominator. Collectively, these estimates demonstrate that $g_{u}\left(P_{u} v, v\right) \geq \frac{1}{4 C_{1}^{4}} g_{u}(v, v) \geq \frac{1}{4 C_{1}^{6}}\|v\|^{2}$ for all $v \in T_{x y}$. At the end, $C_{4}$ can be chosen to be the largest of the constants above.

Returning to the situation of Proposition 3.1, consider another simple Finsler metric $\tilde{F}$ on $\bar{B}$ with corresponding distance function $\tilde{\ell}$.

Proposition 4.4. There exists a constant $C>1$, such that for arbitrary $a \in[0,1]$ and all $x \neq y \in S^{n-1}$ with $\|x-y\| \leq 1 / C$, there holds

$$
\left|\left(d_{1} d_{2}((1-a) \ell+a \tilde{\ell})(x, y)\right)^{n-1}\right| \leq \frac{C}{\|x-y\|^{n-3}}\left|(d x \wedge d y)^{n-1}\right| .
$$

Proof. Given $x, y \in S^{n-1},-y \neq x \neq y$, let $e_{1}, \ldots, e_{n-2}$ be a basis of Euclidean unit vectors of $T_{x y}$, such that $\left(e_{1}, \ldots, e_{n-2}, e_{x y}\right)$ and $\left(e_{1}, \ldots, e_{n-2},-e_{y x}\right)$ form an oriented orthonormal basis of $T_{x} S^{n-1}$ and $T_{y} S^{n-1}$, respectively. Then $d_{1} d_{2} \ell(x, y)^{n-1}=\operatorname{det} A \cdot(d x \wedge d y)^{n-1}$, where the coefficient matrix $A \in \mathbb{R}^{(n-1) \times(n-1)}$ has block structure:

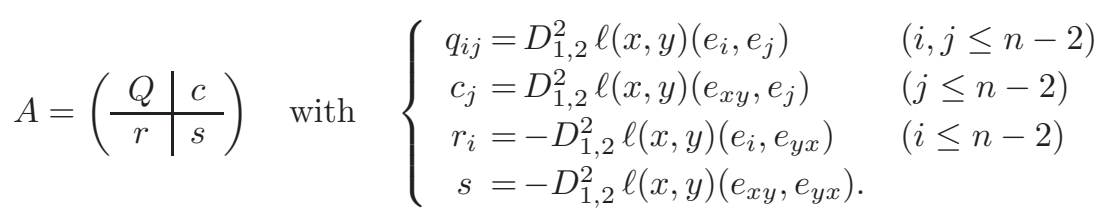

Next, suppose that $\|x-y\| \leq 1 / C_{2}$. Then Lemma 4.2, and inequalities (4.1) and (4.2) imply

$$
\left|D_{1,2}^{2} \ell(x, y)(v, w)+\frac{g_{u}\left(P_{u} v, w\right)}{\ell(x, y)}\right| \leq C_{1}^{2} C_{2}\|v\| \cdot\|w\| \quad \forall v \in T_{x} \bar{B}, w \in T_{y} \bar{B} .
$$


Hence, the difference between the matrices $\frac{-1}{\ell(x, y)}\left(g_{u}\left(P_{u} e_{i}, e_{j}\right)\right)_{i, j}$ and $Q$ is bounded by $C_{1}^{2} C_{2}$. According to (4.1), the matrix $\left(g_{u}\left(P_{u} e_{i}, e_{j}\right)\right)_{i, j}$ in turn is bounded from above by $C_{1}^{2}$. Thus, due to $(4.2)$,

$$
\|Q\| \leq \frac{\left\|\left(g_{u}\left(P_{u} e_{i}, e_{j}\right)\right)_{i, j}\right\|}{\ell(x, y)}+C_{1}^{2} C_{2} \leq \frac{C_{1}^{3}}{\|x-y\|}+C_{1}^{2} C_{2} .
$$

On the other hand, if $\|x-y\| \leq 1 / C_{4}$, Lemma 4.3 states that $g_{u}\left(P_{u} v, v\right)$ is also bounded from below by $\|v\|^{2} / C_{4}$ for all $v \in T_{x y}$. Hence, if $\|x-y\| \leq \frac{1}{2 C_{1}^{3} C_{2} C_{4}}$,

$$
\begin{aligned}
v^{T} Q v & \geq \frac{\|v\|^{2}}{C_{4} \ell(x, y)}-C_{1}^{2} C_{2}\|v\|^{2} \geq \frac{\|v\|^{2}}{C_{1} C_{4}\|x-y\|}-C_{1}^{2} C_{2}\|v\|^{2} \\
& \geq \frac{\|v\|^{2}}{2 C_{1} C_{4}\|x-y\|} \quad \forall v \in \mathbb{R}^{n-2} .
\end{aligned}
$$

Likewise, if $\|x-y\| \leq 1 / C_{2}$, Lemma 4.2 together with the Cauchy inequality and (4.2) show that, for $w \in T_{y} S^{n-1}$,

$$
\begin{aligned}
\left|D_{1,2}^{2} \ell(x, y)\left(e_{x y}, w\right)\right| & \leq \frac{\left|g_{u}\left(P_{u} e_{x y}, w\right)\right|+C_{2}\|x-y\| \cdot\|w\| \sqrt{g_{u}\left(P_{u} e_{x y}, e_{x y}\right)}}{\ell(x, y)} \\
& \leq C_{1} \sqrt{g_{u}\left(P_{u} e_{x y}, e_{x y}\right)}\left(\frac{\sqrt{g_{u}\left(P_{u} w, w\right)}}{\|x-y\|}+C_{2}\|w\|\right) .
\end{aligned}
$$

When $w=e_{j}$ and $\|x-y\| \leq 1 / C_{4}$, one infers from Lemma 4.3 and (4.1) that

$$
\left|c_{j}\right| \leq C_{1} \sqrt{C_{4}}\|x-y\|\left(\frac{C_{1}\left\|e_{j}\right\|}{\|x-y\|}+C_{2}\right) \leq C_{1} \sqrt{C_{4}}\left(C_{1}+\frac{C_{2}}{C_{4}}\right) .
$$

Thus $\|c\| \leq \sqrt{n-2} C_{1} \sqrt{C_{4}}\left(C_{1}+C_{2} / C_{4}\right)$, and the same estimate holds for $\|r\|$, too, since $\ell$ is symmetric upon switching $x$ with $y$. Also, setting $w=-e_{y x}$ and using Lemma 4.3 again, one obtains:

$$
|s| \leq C_{1} \sqrt{C_{4}}\|x-y\|\left(\frac{\sqrt{C_{4}}\|x-y\|}{\|x-y\|}+C_{2}\right)=C_{1}\left(C_{4}+C_{2} \sqrt{C_{4}}\right)\|x-y\| .
$$

After possibly taking larger constants, estimates similar to (4.5)-(4.8) hold true for the entries of $\tilde{A}$ corresponding to $\tilde{\ell}$, and even for the convex combination $\bar{A}:=(1-a) A+a \tilde{A}$ and its submatrices $\bar{Q}, \bar{c}, \bar{r}$ and $\bar{s}$. Especially, (4.6) states the claimed lower estimate for $D_{1,2}^{2} \bar{\ell}(x, y)$ on $T_{x y}$. Now

$$
\left(d_{1} d_{2}((1-a) \ell+a \tilde{\ell})(x, y)\right)^{n-1}=\operatorname{det} \bar{A} \cdot(d x \wedge d y)^{n-1} .
$$

As $\bar{Q}$ is invertible for $\|x-y\|$ sufficiently small, $\operatorname{det} \bar{A}$ can be computed via

$$
\operatorname{det} \bar{A}=\operatorname{det}\left(\begin{array}{c|c}
\bar{Q} & 0 \\
\hline 0 & 1
\end{array}\right) \operatorname{det}\left(\begin{array}{c|c}
1 & \bar{Q}^{-1} \bar{c} \\
\hline \bar{r} & \bar{s}
\end{array}\right)=\operatorname{det} \bar{Q} \cdot\left(\bar{s}-\bar{r} \bar{Q}^{-1} \bar{c}\right),
$$

e.g., by Laplace expansion along the last row. 
Furthermore, one infers from (4.5) and (4.6) that

$$
\operatorname{det} \bar{Q} \leq\left(\frac{2 C_{1}^{3}}{\|x-y\|}\right)^{n-2} \text { and }\left\|\bar{Q}^{-1}\right\| \leq 2 C_{1} C_{4}\|x-y\| .
$$

Combining the above estimates, (4.9) implies for $\|x-y\|<\frac{1}{2 C_{1}^{3} C_{2} C_{4}}$ :

$$
|\operatorname{det} \bar{A}| \leq|\operatorname{det} \bar{Q}| \cdot\left(|\bar{s}|+\|\bar{r}\| \cdot\|\bar{c}\| \cdot\left\|\bar{Q}^{-1}\right\|\right) \leq \frac{C}{\|x-y\|^{n-3}}
$$

for some constant $C$, thereby proving the assertion.

Remarks. 1) The estimates in the proof also yield a sufficient condition for the nonvanishing of $\hat{\eta}=n \cdot \int_{0}^{1}\left(d d_{2}((1-a) \ell+a \tilde{\ell})\right)^{n-1} d a$. Namely, assume for $\|x-y\|<$ $\varepsilon:=1 /\left(2 C_{1}^{3} C_{2} C_{4}\right)$, that

$$
D_{1,2}^{2}(\tilde{\ell}-\ell)(x, y)(v, v) \leq \frac{\varepsilon\|v\|^{2}}{2 C_{1} C_{4}\|x-y\|} \quad \forall v \in T_{x y} .
$$

Here, $C_{1}, C_{2}$ and $C_{4}$ are the constants related to $\ell$ as before. Then, with the above notations, $(1-a) Q+a \tilde{Q}$ is non-degenerate on $T_{x y}$, for all $a \in[0,1]$ and $\|x-y\| \leq \varepsilon$. Further, $\left(d d_{2}((1-a) \ell+a \tilde{\ell})\right)^{n-1}(x, y)=0$, if and only if

$$
0=\frac{\operatorname{det} \bar{A}}{\operatorname{det} \bar{Q}}=s+a(\tilde{s}-s)-(r+a(\tilde{r}-r))(Q+a(\tilde{Q}-Q))^{-1}(c+a(\tilde{c}-c)) .
$$

Since $\left(d_{1} d_{2} \ell\right)^{n-1}$ is non-degenerate, $0 \neq \operatorname{det} A$ and thus $0 \neq s-r Q^{-1} c$. Therefore, one could deduce bounds on $|\tilde{s}-s|,\|\tilde{r}-r\|$, and $\|\tilde{c}-c\|$, that would guarantee $\operatorname{det}((1-a) A+a \tilde{A}) \neq 0$ for all $a \in[0,1]$ and $\|x-y\| \leq \varepsilon$.

2 ) In the model case of the Euclidean metric on $\bar{B}$, it follows from

$$
D^{2} \ell(x, y)(v, w)=-\frac{\langle v, w\rangle-\langle v, u\rangle \cdot\langle u, w\rangle}{\|x-y\|}, \quad u=\frac{y-x}{\|y-x\|}, \quad e_{x y}=\frac{y-\langle x, y\rangle x}{\sqrt{1-\langle x, y\rangle^{2}}}
$$

that $Q=-\|x-y\|^{-1} \cdot \mathbf{1}, r=c^{T}=(0, \ldots, 0)$ and $s=\|x-y\| / 4$. This example might suggest, that $\left|s-r Q^{-1} c\right| \geq\|x-y\| / C^{\prime}$ should hold in general for some $C^{\prime}>1$ and $\|x-y\|<\varepsilon$. However, the estimates from Lemmas 4.2 and 4.3 are too weak to verify this conjecture, since the error term is of the same order.

The next corollary fills a gap in the proof of Proposition 3.1.

Corollary 4.5. $\left(d d_{2}((1-a) \ell+a \tilde{\ell})\right)^{n-1}$ is integrable on $\Pi$ for all $a \in[0,1]$.

Proof. For continuity in the interior of $\Pi$, it is sufficient to verify integrability in a neighbourhood of the diagonal. To this end, let $z_{k}=y_{k}-x_{k}$; hence $\left(x_{1}, \ldots, x_{n}, z_{1}, \ldots, z_{n}\right)$ are new coordinates on $\mathbb{R}^{n} \times \mathbb{R}^{n}$, and the diagonal is just $\{(x, z): z=0\}$. Further, $(d x \wedge d y)^{n-1}=(d x \wedge d z)^{n-1}$ plus a term that involves $(d x)^{n}$ and thus vanishes after restriction to $S^{n-1} \times S^{n-1}$. In the new coordinates, 
$S^{n-1} \times S^{n-1}=\left\{(x, z): x \in S^{n-1}, z \in S^{n-1}-x\right\}$, where $S^{n-1}-x$ is the sphere translated by $-x$. One can switch from $z$ to polar-like coordinates $\left(r, \theta_{1}, \ldots, \theta_{n-2}\right)$, with $r=\|z\|$ and local angle coordinates $\left(\theta_{1}, \ldots, \theta_{n-2}\right)$ on $S_{r}^{n-1} \cap\left(S^{n-1}-x\right)$. From the transformation formula, there is a coefficient function $c=c(\theta)$ such that $(d z)^{n-1}=c(\theta) \cdot r^{n-2} d r \wedge(d \theta)^{n-2}$ on $S^{n-1}-x$. Since $r=\|x-y\|$, one infers from Proposition 4.4 that

$$
\begin{aligned}
\left|\left(d_{1} d_{2}((1-a) \ell+a \tilde{\ell})(x, y)\right)^{n-1}\right| & \leq \frac{C}{\|x-y\|^{n-3}}\left|(d x \wedge d y)^{n-1}\right| \\
& =C \cdot r\left|c(\theta) d r \wedge(d \theta)^{n-2} \wedge(d x)^{n-1}\right|
\end{aligned}
$$

holds for $(x, y) \in \Pi$ with $\|x-y\|<1 / C$.

\section{A counterexample for positivity of $\hat{\eta}$}

One can ask whether $\hat{\eta}$ (as defined in Proposition 3.1) is always a volume form in the given situation. Unfortunately, this is wrong.

Proposition 5.1. There are simple Riemannian metrics, such that induced distances $\ell$ and $\tilde{\ell}$ satisfy $\tilde{\ell}(y, z) \geq \ell(y, z)$ for all $y, z \in S^{n-1}$, but such that $\hat{\eta}$ is indefinite and there is no simple Finsler metric with boundary distance $\tilde{\ell}+\ell$.

Proof (by construction). Let $\ell$ be the Euclidean distance on $\bar{B} \subset \mathbb{R}^{3}$. Take $y=e_{3}=(0,0,1), z=-e_{3}, v \in T_{y} \partial B$ and $w \in T_{z} \partial B$. Using $v \perp e_{3} \perp w$, one obtains:

$$
\begin{aligned}
d d_{2} \ell(y, z)(v+0,0+w) & =\left.\frac{d^{2}}{d s d t}\right|_{s=t=0}\|y+s v-z-t w\| \\
& =\left.\frac{d}{d t}\right|_{0} \frac{\langle v, y-z-t w\rangle}{\|y-z-t w\|}=-\frac{\langle v, w\rangle}{2}
\end{aligned}
$$

Further, let $\varphi: \bar{B} \rightarrow \bar{B}$ be a diffeomorphism with $\varphi(y)=y$ and $\varphi(z)=z$, and consider the metric $\tilde{\ell}:=r \varphi^{*} \ell$ for some constant $r>1$. Since $\tilde{\ell}$ is induced by the flat Riemannian metric $r^{2} \varphi^{*}\langle\cdot, \cdot\rangle,(\bar{B}, \tilde{\ell})$ is still simple, and for $v, w \perp e_{3}$

$$
\begin{aligned}
d d_{2} \tilde{\ell}(y, z)(v+0,0+w) & =\left.\frac{d^{2}}{d s d t}\right|_{s=t=0} r\|\varphi(y+s v)-\varphi(z-t w)\| \\
& =\left.r \frac{d}{d t}\right|_{0} \frac{\langle D \varphi(y) v, y-\varphi(z-t w)\rangle}{\|y-\varphi(z-t w)\|}=-r \frac{\langle D \varphi(y) v, D \varphi(z) w\rangle}{2} .
\end{aligned}
$$

Let $A, \mathbf{1} \in \mathbb{R}^{2 \times 2}$ denote the matrices of $D \varphi(y)^{T} D \varphi(z)$ with respect to $e_{1}$ and $e_{2}$, and the identity, respectively. The evaluation of $\hat{\eta}(y, z)$ on the basis of $T_{(y, z)} M \simeq$ 
$T_{y} \partial B \oplus T_{z} \partial B$ given by $b_{1}=e_{1}+0, b_{2}=0+e_{1}, b_{3}=e_{2}+0, b_{4}=0+e_{2}$ yields

$$
\begin{aligned}
\hat{\eta}(y, z) & \left(b_{1}, b_{2}, b_{3}, b_{4}\right) \\
& =\frac{1}{2}\left(d d_{2} \ell(y, z)^{2}+d d_{2}(\ell+\tilde{\ell})(y, z)^{2}+d d_{2} \tilde{\ell}(y, z)^{2}\right)\left(b_{1}, b_{2}, b_{3}, b_{4}\right) \\
& =\frac{1}{2}\left(\operatorname{det}\left(\frac{1}{2} \mathbf{1}\right)+\operatorname{det}\left(\frac{1}{2} \mathbf{1}+\frac{r}{2} A\right)+\operatorname{det}\left(\frac{r}{2} A\right)\right) \\
& =\frac{1}{8}\left(2+r \cdot \operatorname{tr}(A)+2 r^{2} \operatorname{det}(A)\right) .
\end{aligned}
$$

In order to get a negative result, $A$ should have two negative eigenvalues of different magnitudes, so as to get a large negative trace and a comparatively small, though positive determinant. A possible way to construct $\varphi$ with such $A$ is to compose $\varphi$ by stretching the ball near $y$ and $z$ with reciprocal factors and U-turntorsion around the $e_{3}$-axis.

Therefore, consider the two parametrizations

$$
\psi_{ \pm}: \mathbb{R}^{2} \rightarrow S^{2} \cap\left\{ \pm x_{3}>0\right\}, \quad \psi_{ \pm}(\xi)=\frac{1}{\sqrt{1+\|\xi\|^{2}}}\left(\begin{array}{c}
\xi_{1} \\
\xi_{2} \\
\pm 1
\end{array}\right)
$$

of the upper and lower hemisphere. Further, set $\rho(t)=\exp \left(-s^{2} t^{2} / 2\right)$ for $s>1$ fixed and define maps $\phi_{ \pm}: \mathbb{R}^{2} \rightarrow \mathbb{R}^{2}$ via

$$
\phi_{ \pm}(\xi)=\left(\begin{array}{c}
\xi_{1} \mp \rho\left(\xi_{2}\right) \xi_{2} / s \\
\xi_{2} \pm s \rho\left(\xi_{1}\right) \xi_{1}
\end{array}\right)
$$

Finally, set $\varphi(x):=\|x\| \cdot \psi_{ \pm}^{-1} \circ \phi_{ \pm} \circ \psi_{ \pm}(x /\|x\|)$ for $x_{3} \neq 0$ and $\varphi(x)=x$ otherwise. Notice that $\varphi$ is differentiable along the equator, since $\psi_{ \pm}^{-1}(x)=\frac{ \pm 1}{x_{3}}\left(\begin{array}{l}x_{1} \\ x_{2}\end{array}\right)$ and $\exp \left(-s^{2} x_{1,2}^{2} / 2 x_{3}^{2}\right)$ decays rapidly as $\left|x_{3}\right| \rightarrow 0$. The differential of $\phi_{ \pm}$is

$$
D \phi_{ \pm}(\xi)=\left(\begin{array}{cc}
1 & \mp\left(1-s^{2} \xi_{2}^{2}\right) \rho\left(\xi_{2}\right) / s \\
\pm s\left(1-s^{2} \xi_{1}^{2}\right) \rho\left(\xi_{1}\right) & 1
\end{array}\right)
$$

with $\operatorname{det}\left(D \phi_{ \pm}(\xi)\right)=1+\left(1-s^{2} \xi_{1}^{2}\right) \rho\left(\xi_{1}\right)\left(1-s^{2} \xi_{2}^{2}\right) \rho\left(\xi_{2}\right)$. Also, as follows from $\frac{d}{d t}(1-t) e^{-t / 2}=\frac{t-3}{2} e^{-t / 2}=0 \Leftrightarrow t=3$, the coefficients $\left(1-s^{2} \xi_{i}^{2}\right) \rho\left(\xi_{i}\right)$ range between $-2 e^{-3 / 2}$ and 1 ; so $\operatorname{det}\left(D \phi_{ \pm}(\xi)\right) \geq 1-2 e^{-3 / 2}>1 / 2$. Consequently $\phi_{ \pm}$ are diffeomorphism, and thus $\varphi$ is also a diffeomorphism outside the origin, where it could be smoothed without loss of the boundary distance estimate.

Due to $D \psi_{ \pm}\left( \pm e_{3}\right)=\mathbf{1}$, the matrix $A$ related to the specified $\varphi$ is

$$
A=D \phi_{+}(0)^{T} D \phi_{-}(0)=\left(\begin{array}{cc}
1-s^{2} & s+1 / s \\
-s-1 / s & 1-1 / s^{2}
\end{array}\right)
$$

with $\operatorname{tr} A=2-s^{2}-s^{-2}$ and $\operatorname{det} A=4$. For $\hat{\eta}(y, z)\left(b_{1}, b_{2}, b_{3}, b_{4}\right)$ to be negative, it is then necessary that

$$
0>\frac{1}{8}\left(2+r \cdot \operatorname{tr}(A)+2 r^{2} \operatorname{det}(A)\right)=\frac{1}{8}\left(2+r\left(2-s^{2}-s^{-2}\right)+8 r^{2}\right),
$$

whereas $r$ must also match $s$ to guarantee that $r \varphi^{*} \ell>\ell$. This in turn will hold, provided that $r\left\|D\left(\psi_{ \pm} \circ \phi_{ \pm}\right)(\xi) v\right\| \geq\left\|D \psi_{ \pm}(\xi) v\right\|$ for all $\xi, v \in \mathbb{R}^{2}$. 
Therefore, one computes

$$
\begin{aligned}
& \left\|D \psi_{ \pm}(\xi) v\right\|^{2}=\left\|\left.\frac{d}{d t}\right|_{t=0} \psi_{ \pm}(\xi+t v)\right\|^{2}=\frac{\|v\|^{2}}{1+\|\xi\|^{2}}-\frac{\langle v, \xi\rangle^{2}}{\left(1+\|\xi\|^{2}\right)^{2}} \\
& \text { so } \quad\left\|D\left(\psi_{ \pm} \circ \phi_{ \pm}\right)(\xi) v\right\|^{2}=\frac{\left\|D \phi_{ \pm}(\xi) v\right\|^{2}}{1+\left\|\phi_{ \pm}(\xi)\right\|^{2}}+\frac{\left\langle D \phi_{ \pm}(\xi) v, \phi_{ \pm}(\xi)\right\rangle^{2}}{\left(1+\left\|\phi_{ \pm}(\xi)\right\|^{2}\right)^{2}}
\end{aligned}
$$

Applying $(a+b)^{2} \leq 2 a^{2}+2 b^{2}$ and the triangle inequality gives

$$
\begin{aligned}
\left\|\phi_{ \pm}(\xi)\right\|^{2} & \leq\left(\left\|\left(\begin{array}{c}
\xi_{1} \\
\pm s \xi_{1} \rho\left(\xi_{1}\right)
\end{array}\right)\right\|+\left\|\left(\begin{array}{c}
\mp \xi_{2} \rho\left(\xi_{2}\right) / s \\
\xi_{2}
\end{array}\right)\right\|\right)^{2} \\
& \leq 2 \xi_{1}^{2}\left(1+s^{2} \rho\left(\xi_{1}\right)^{2}\right)+2 \xi_{2}^{2}\left(1+\rho\left(\xi_{2}\right)^{2} / s^{2}\right) \\
& \leq 2\|\xi\|^{2}+2 s^{2} \xi_{1}^{2} \rho\left(\xi_{1}\right)^{2}+2 s^{2} \xi_{2}^{2} \rho\left(\xi_{2}\right)^{2} \leq 2\|\xi\|^{2}+4
\end{aligned}
$$

because of $s^{2} \xi_{i}^{2} \rho\left(\xi_{i}\right)^{2} \leq \frac{s^{2} \xi_{i}^{2}}{1+s^{2} \xi_{i}^{2}}<1$. This yields for the quotient of the denominators the bound

$$
\frac{1+\left\|\phi_{ \pm}(\xi)\right\|^{2}}{1+\|\xi\|^{2}} \leq \frac{5+2\|\xi\|^{2}}{1+\|\xi\|^{2}} \leq 5
$$

It remains to estimate the numerators. In the sequel, vectors are interpreted as single-column matrices; e.g., $\langle v, w\rangle$ becomes $v^{T} w$. Then for $\xi \in \mathbb{R}^{2}$ fixed,

$$
\begin{aligned}
q(\xi) & :=\sup _{v \in \mathbb{R}_{*}^{2}} \frac{\|v\|^{2}-\left(1+\|\xi\|^{2}\right)^{-1}\langle v, \xi\rangle}{\left\|D \phi_{ \pm}(\xi) v\right\|^{2}-\left(1+\left\|\phi_{ \pm}(\xi)\right\|^{2}\right)^{-1}\left\langle D \phi_{ \pm}(\xi) v, \phi_{ \pm}(\xi)\right)^{2}} \\
& =\sup _{v \in \mathbb{R}_{*}^{2}} \frac{v^{T}\left(\mathbf{1}-\left(1+\|\xi\|^{2}\right)^{-1} \xi \xi^{T}\right) v}{v^{T} D \phi_{ \pm}(\xi)^{T}\left(\mathbf{1}-\left(1+\left\|\phi_{ \pm}(\xi)\right\|^{2}\right)^{-1} \phi_{ \pm}(\xi) \phi_{ \pm}(\xi)^{T}\right) D \phi_{ \pm}(\xi) v} .
\end{aligned}
$$

Since $\left(\mathbf{1}-\left(1+\|w\|^{2}\right) w w^{T}\right)^{-1}=\mathbf{1}+w w^{T}$ is positive and symmetric for all $w \in \mathbb{R}^{3}$, it has a unique positive, symmetric square root. When substituting $v=D \phi_{ \pm}(\xi)^{-1}$. $\sqrt{\mathbf{1}+\phi_{ \pm}(\xi) \phi_{ \pm}(\xi)^{T}} u$, one obtains

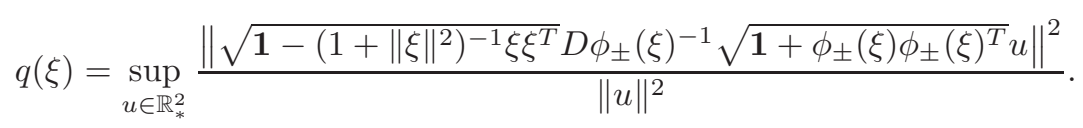

Writing $B(\xi)$ for the operator in the numerator, this is just the largest eigenvalue of $B(\xi)^{T} B(\xi)$. It can be majorized by its trace, and using the invariance of traces under cyclic permutation and linearity gives

$$
\begin{aligned}
q(\xi)< & \operatorname{tr}\left(B(\xi)^{T} B(\xi)\right) \\
= & \operatorname{tr}\left(D \phi_{ \pm}(\xi)^{-T}\left(\mathbf{1}-\left(1+\|\xi\|^{2}\right)^{-1} \xi \xi^{T}\right) D \phi_{ \pm}(\xi)^{-1}\left(\mathbf{1}+\phi_{ \pm}(\xi) \phi_{ \pm}(\xi)^{T}\right)\right) \\
= & \operatorname{tr}\left(D \phi_{ \pm}(\xi)^{-T} D \phi_{ \pm}(\xi)^{-1}\right)-\left(1+\|\xi\|^{2}\right)^{-1} \operatorname{tr}\left(D \phi_{ \pm}(\xi)^{-T} \xi \xi^{T} D \phi_{ \pm}(\xi)^{-1}\right) \\
& +\operatorname{tr}\left(D \phi_{ \pm}(\xi)^{-T}\left(\mathbf{1}-\left(1+\|\xi\|^{2}\right)^{-1} \xi \xi^{T}\right) D \phi_{ \pm}(\xi)^{-1} \phi_{ \pm}(\xi) \phi_{ \pm}(\xi)^{T}\right) \\
\leq & \operatorname{tr}\left(D \phi_{ \pm}(\xi)^{-T} D \phi_{ \pm}(\xi)^{-1}\right) \\
& +\phi_{ \pm}(\xi)^{T} D \phi_{ \pm}(\xi)^{-T}\left(\mathbf{1}-\left(1+\|\xi\|^{2}\right)^{-1} \xi \xi^{T}\right) D \phi_{ \pm}(\xi)^{-1} \phi_{ \pm}(\xi)
\end{aligned}
$$


Because $D \phi_{ \pm}(\xi)^{-1}=\operatorname{det}\left(D \phi_{ \pm}(\xi)\right)^{-1} D \phi_{\mp}(\xi)$, the first summand reads

$$
\begin{aligned}
\operatorname{tr}\left(D \phi_{ \pm}(\xi)^{-T} D\right. & \left.\phi_{ \pm}(\xi)^{-1}\right)=\frac{\operatorname{tr}\left(D \phi_{\mp}(\xi)^{T} D \phi_{\mp}(\xi)\right)}{\operatorname{det}\left(D \phi_{ \pm}(\xi)\right)^{2}} \\
& =\frac{2+s^{2}\left(1-s^{2} \xi_{1}^{2}\right)^{2} \rho\left(\xi_{1}\right)^{2}+s^{-2}\left(1-s^{2} \xi_{2}^{2}\right)^{2} \rho\left(\xi_{2}\right)^{2}}{\left(1+\left(1-s^{2} \xi_{1}^{2}\right) \rho\left(\xi_{1}\right)\left(1-s^{2} \xi_{2}^{2}\right) \rho\left(\xi_{2}\right)\right)^{2}}<4\left(s^{2}+3\right)
\end{aligned}
$$

due to $-1 / 2<\left(1-s^{2} \xi_{i}^{2}\right) \rho\left(\xi_{i}\right) \leq 1$, as stated before. Further, one can apply

$$
\mathbf{1}-\left(1+\|\xi\|^{2}\right)^{-1} \xi \xi^{T}=\frac{1+J \xi(J \xi)^{T}}{1+\|\xi\|^{2}}, \quad \text { with } J=\left(\begin{array}{cc}
0 & -1 \\
1 & 0
\end{array}\right)
$$

to rewrite the second summand and obtain

$$
q(\xi)<4\left(s^{2}+3+\frac{\left\|D \phi_{\mp}(\xi) \phi_{ \pm}(\xi)\right\|^{2}+\left\langle J \xi, D \phi_{\mp}(\xi) \phi_{ \pm}(\xi)\right\rangle^{2}}{\left(1+\|\xi\|^{2}\right)}\right) .
$$

Now,

$$
D \phi_{\mp}(\xi) \phi_{ \pm}(\xi)=\left(\begin{array}{c}
\xi_{1}+\xi_{1}\left(1-s^{2} \xi_{2}^{2}\right) \rho\left(\xi_{1}\right) \rho\left(\xi_{2}\right) \\
\xi_{2}+\xi_{2}\left(1-s^{2} \xi_{1}^{2}\right) \rho\left(\xi_{1}\right) \rho\left(\xi_{2}\right)
\end{array}\right)+\left(\begin{array}{c}
-s \xi_{2}^{3} \rho\left(\xi_{2}\right)^{2} \\
s^{3} \xi_{1}^{3} \rho\left(\xi_{1}\right)^{2}
\end{array}\right) .
$$

Because $\frac{d}{d t} t^{m} \rho(t)=\left(m-s^{2} t^{2}\right) t^{m-1} \rho(t)$ vanishes for $(t=0$ and $) t^{2}=m s^{-2}$, the functions $\xi_{i}^{m} \rho\left(\xi_{i}\right)$ have their maxima at $(m / e)^{m / 2} s^{-m}$. Hence, the triangle inequality gives

$$
\left\|D \phi_{\mp}(\xi) \phi_{ \pm}(\xi)\right\| \leq 2\|\xi\|+\left(\frac{3}{2 e}\right)^{3 / 2} \sqrt{1+s^{-2}}<2\|\xi\|+1,
$$

and $(a+b)^{2} \leq 2 a^{2}+2 b^{2}$ implies $\left\|D \phi_{\mp}(\xi) \phi_{ \pm}(\xi)\right\|^{2} \leq 8\|\xi\|^{2}+2$. Also,

$$
\begin{aligned}
\left\langle J \xi, D \phi_{\mp}(\xi) \phi_{ \pm}(\xi)\right\rangle & =s^{2}\left(\xi_{1} \xi_{2}^{3}-\xi_{1}^{3} \xi_{2}\right) \rho\left(\xi_{1}\right) \rho\left(\xi_{2}\right)+s \xi_{2}^{4} \rho\left(\xi_{2}\right)^{2}+s^{3} \xi_{1}^{4} \rho\left(\xi_{1}\right)^{2} \\
& \leq 2 \cdot 3^{3 / 2} e^{-2} s^{-2}+2^{2} e^{-2}\left(s^{-3}+s^{-1}\right)<3 .
\end{aligned}
$$

Assembling these estimates leads to

$$
q(\xi)<4\left(s^{2}+3+\frac{8\|\xi\|^{2}+2+3^{2}}{\left(1+\|\xi\|^{2}\right)}\right)<4\left(s^{2}+14\right)
$$

and shows that

$$
\frac{\left\|D \psi_{ \pm}(\xi) v\right\|}{\left\|D\left(\psi_{ \pm} \circ \phi_{ \pm}\right)(\xi) v\right\|}<10 \sqrt{s^{2}+4}=: r \quad \forall v, \xi \in \mathbb{R}^{2}, v \neq 0 .
$$

Finally, $s$ can be chosen sufficiently large to guarantee that

$$
0>\hat{\eta}(y, z)\left(b_{1}, b_{2}, b_{3}, b_{4}\right)=\frac{1}{8}\left(2+r\left(2-s^{2}-s^{-2}\right)+8 r^{2}\right) .
$$

This also proves that there must not be a simple Finsler metric with boundary distances $\ell+\tilde{\ell}$, because then $\hat{\eta}=\frac{1}{2}\left(d d_{2} \ell\right)^{2}+\frac{1}{2}\left(d d_{2}(\ell+\tilde{\ell})\right)^{2}+\frac{1}{2}\left(d d_{2} \tilde{\ell}\right)^{2}$, as a sum of volume forms, would be positive. 


\section{References}

[1] Burago, D. And Ivanov, S.: Boundary rigidity and filling volume minimality of metrics close to a flat one. Ann. of Math. (2) 171 (2010), 1183-1211.

[2] Burago, D. And Ivanov, S.: Area minimizers and boundary rigidity of almost hyperbolic metrics. Duke Math. J. 162 (2013), no. 7, 1205-1248

[3] Croke, C. B.: Rigidity theorems in riemannian geometry. In Geometric methods in inverse problems and PDE control, 47-72. IMA Vol. Math. Appl. 137, Springer, New York, 2004.

[4] Croke, C. B. and Dairbekov, N. S.: Lengths and volumes in Riemannian manifolds. Duke Math. J. 125 (2004), no. 1, 1-14.

[5] Croke, C. B., Dairbekov, N.S. and Sharafutdinov, V. A.: Local boundary rigidity of a compact Riemannian manifold with curvature bounded above. Trans. Amer. Math. Soc. 352 (2000), no. 9, 3937-3956.

[6] Gromov, M.: Filling Riemannian manifolds. J. Differential Geom. 18 (1983), 1-147.

[7] Ivanov, S.: Filling minimality of Finslerian 2-discs. Proc. Steklov Inst.Math. 273 (2011), 176-190.

[8] Ivanov, S.: Local monotonicity of Riemannian and Finsler volume with respect to boundary distances. Geom. Dedicata 164 (2013), 83-96.

[9] Oтац, J. P.: Sur la géometrie symplectique de l'espace des géodésiques d'une variété à courbure négative. Rev. Mat. Iberoamericana 8 (1992), no. 3, 441-456.

[10] Shen, Z.: Lectures on Finsler geometry. World Scientific, Singapore, 2001.

Received May 31, 2012.

Henrik Koehler: Fakultät für Mathematik, Ruhr-Universität Bochum, 44780

Bochum, Germany.

E-mail: henrik.koehler@rub.de 